\title{
Filigrane
}

Écoutes psychanalytiques

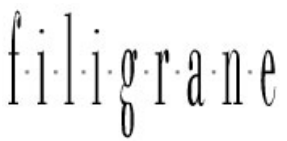

\section{Effet de corps en présence, adolescence, féminin et maternel}

\section{Anne Boisseuil}

Volume 28, numéro 2, 2019

URI : https://id.erudit.org/iderudit/1069699ar

DOI : https://doi.org/10.7202/1069699ar

Aller au sommaire du numéro

\section{Éditeur(s)}

Santé mentale et société

\section{ISSN}

1192-1412 (imprimé)

1911-4656 (numérique)

Découvrir la revue

Citer cet article

Boisseuil, A. (2019). Effet de corps en présence, adolescence, féminin et maternel. Filigrane, 28(2), 169-186. https://doi.org/10.7202/1069699ar

\section{Résumé de l'article}

Alors que l'interdit du toucher est au centre du dispositif psychanalytique, le travail thérapeutique avec des adolescents remobilise de manière singulière la conceptualisation du cadre et du processus. Que dire alors du moment où la thérapeute est enceinte ? La question du corps est au coeur du travail de subjectivation, à la fois sous ses aspects sensoriels et sous ses aspects génitaux. C'est ce que nous travaillerons avec le déroulement de la thérapie d'une jeune adolescente, Mila, chez qui l'image du corps est fragile, attaquée, du dehors et du dedans. Elle le mettra en acte en se mettant en danger, en se marquant le corps par des maquillages, des piercings et par des tenues ambigües quant au genre, des tenues d'animaux... Dessinant beaucoup pendant les séances, nous pourrons étudier les mouvements projectifs de son image du corps ainsi que les éléments sensoriels et sensuels contenus dans le contact avec la feuille. Durant sa thérapie, le fait que j'ai été enceinte a remobilisé chez elle des ancrages archaïques qui lui ont permis de retravailler la constitution de son enveloppe psychique bisexuée. L'attention portée à un corps invisible mais présent engagea un travail hallucinatoire, au sens d'un travail du rêve qui s'est traduit par un rêve rapporté en séance et qui se répètera de manière signifiante au cours de sa thérapie, à une date anniversaire. De même, nous penserons à l'effet révélateur, à l'occasion de mon arrêt, de confusions précoces entre les enveloppes psychiques de la mère et de la jeune fille.
Ce document est protégé par la loi sur le droit d'auteur. L’utilisation des services d’Érudit (y compris la reproduction) est assujettie à sa politique d'utilisation que vous pouvez consulter en ligne.

https://apropos.erudit.org/fr/usagers/politique-dutilisation/ 


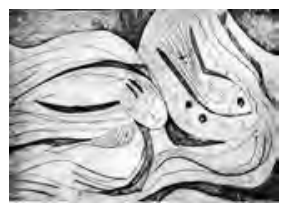

\title{
Effet de corps en présence, adolescence, féminin et maternel
}

\author{
Anne Boisseuil
}

\begin{abstract}
Résumé: Alors que l'interdit du toucher est au centre du dispositif psychanalytique, le travail thérapeutique avec des adolescents remobilise de manière singulière la conceptualisation du cadre et du processus. Que dire alors du moment où la thérapeute est enceinte? La question du corps est au cœur du travail de subjectivation, à la fois sous ses aspects sensoriels et sous ses aspects génitaux. C'est ce que nous travaillerons avec le déroulement de la thérapie d'une jeune adolescente, Mila, chez qui l'image du corps est fragile, attaquée, du dehors et du dedans. Elle le mettra en acte en se mettant en danger, en se marquant le corps par des maquillages, des piercings et par des tenues ambigües quant au genre, des tenues d'animaux... Dessinant beaucoup pendant les séances, nous pourrons étudier les mouvements projectifs de son image du corps ainsi que les éléments sensoriels et sensuels contenus dans le contact avec la feuille. Durant sa thérapie, le fait que j'ai été enceinte a remobilisé chez elle des ancrages archaïques qui lui ont permis de retravailler la constitution de son enveloppe psychique bisexuée. L'attention portée à un corps invisible mais présent engagea un travail hallucinatoire, au sens d'un travail du rêve qui s'est traduit par un rêve rapporté en séance et qui se répètera de manière signifiante au cours de sa thérapie, à une date anniversaire. De même, nous penserons à l'effet révélateur, à l'occasion de mon arrêt, de confusions précoces entre les enveloppes psychiques de la mère et de la jeune fille.
\end{abstract}

Mots clés: corps; grossesse; transfert; adolescence; bisexualité précoce.

\begin{abstract}
While the prohibition of touch is at the center of the psychoanalytic device, the therapeutic work with adolescents singularly remobilizes the conceptualization of its framework and process. What to say then when the therapist is pregnant? The question of the body is at the heart of the work of subjectivation, both in its sensory and genital aspects. This is what we will work on in this paper by analyzing the course of a therapy with a young teenager, Mila, in whom the image of the body is fragile, attacked from outside and from within. She will put it into action by putting herself in danger, by marking the body with make-up, piercings and presentations of outfits that are ambiguous as to the gender, animal outfits, etc. Since she was drawing a lot during the sessions, we were able to study the projective movements of her image of the body as well as sensory and sensory elements contained in the contact with the paper. During her therapy, the fact that I was pregnant remobilized her archaic anchors and allowed her to rework the constitution of her bisexual psychic envelope. The attention paid to an invisible but present
\end{abstract}


body led to a hallucinatory work, in the sense of a dream work that resulted in a dream reported in a session and repeated in a significant way during her therapy, on an anniversary date. In the same way, we will think about the revealing effect, at the time of my maternity leave, of early confusions between the psychic envelopes of the mother and the girl.

Key words: body; pregnancy; transfer; adolescence; early bisexuality.

e corps en séance a été mis au silence par le dispositif divan/fauteuil, — tel que l'a mis en place Freud dans le but d'épargner à l'analysant «tout effort musculaire, toute impression sensorielle, capable de détourner son attention de sa propre activité psychique» (Freud, 1904, p. 3). Ce dispositif s'est trouvé rapidement mis en question par les contemporains de Freud, dont S. Ferenczi (1931) et M. Klein (1932) qui ont développé une écoute de l'activité psychique à la fois par les associations verbales et les affects, mais aussi les actions corporelles, comme dans le jeu avec les enfants. Cette écoute implique donc naturellement beaucoup plus le corps de l'analyste puisqu'il est mobilisé dans toute sa "caisse de résonance» sensorielle. Cet engagement sensori-moteur participe à l'évolution du contre-transfert, celui-ci n'étant pas seulement une réaction défensive à l'égard du patient, mais aussi le support ou le contenant d'éléments non subjectivés ou projetés. J. Bleger (1979) propose que le cadre thérapeutique soit le lieu de « dépôt » des parties les moins différenciées du soi, celles qui font corps avec l'objet. Nous interrogerons cette conception du cadre lorsque la thérapeute est enceinte. Alors que tout le dispositif analytique selon la pensée freudienne tend à la discrétion du corps du thérapeute, qu'en est-il d'une thérapeute qui témoigne, par son corps, d'une vie intime qui exclut le patient? En quoi cela participe-t-il au processus thérapeutique?

Le courant de pensée sur la symbolisation primaire (Golse et Roussillon, 2010) s'ancre sur les sensations perçues et celles représentées, où la nécessité d'un effacement de la présence de l'analyste n'est plus. En effet, seront analysés les effets de présence et l'importance du réel du sexe de l'analyste, de son corps, de ses manifestations visibles, quand tout le champ non verbal de sa communication devient source de potentielle transitionnalité et de développement du soi du patient. Porter attention au corps revient aussi à porter attention à toutes les identifications primaires contenues dans cette enveloppe corporelle. Freud (1911) désigne l'attention comme un processus actif qui permet de construire les premiers sentiments du moi. L'attention que la thérapeute peut porter aux effets de sa grossesse sur le processus 
thérapeutique serait donc déterminante dans la reconnaissance de la remobilisation d'identifications précoces du patient avec certains aspects de la situation thérapeutique. En effet, un autre corps est présent, plus ou moins discret, mais fondamentalement là. Les identifications inconscientes au bébé ne sont pas les seules; il ne s'agit pas seulement d'une mobilisation de fantasmes et de représentations, mais aussi d'effets de présence; celle du bébé réel dans la séance, celle d'une femme enceinte, celle d'une séparation annoncée.

Dans le cas de patients adolescents, P. Gutton (2009) définit d'autres modalités de compréhension du processus thérapeutique qu'il appelle une «conversation ordinaire» avec l'adolescent. En effet, avec ces derniers, il axe la technique d'écoute thérapeutique sur la relance de l'associativité au sein de la séance et, pour certains adolescents, davantage encore que sur l'interprétation de fantasmes inconscients. Il s'agit d'un transfert sur la séance et/ ou sur le thérapeute. Cette conception de la thérapie s'inscrit dans un changement paradigmatique où ce sont les mécanismes de construction de la pensée qui sont au centre de l'attention du thérapeute. Il s'intéresse à des adolescents chez qui l'activité fantasmatique est entravée et le fonctionnement psychique dominé par la projection, ce qu'il nomme «l'extériorisation", où l'autre n'est pas tout à fait un objet total. Selon lui, les blessures du moi de ces adolescents ne leur permettent pas d'intérioriser des interprétations. L'espace de la séance peut devenir alors le contenant d'une associativité co-construite entre le thérapeute et l'adolescent, une "ex-timité». Le jeune patient demande d'abord à être reconnu avant d'être compris et cela passe aussi par la reconnaissance de l'effet du corps de l'autre.

Les effets dans la thérapie de la gravidité de la thérapeute font l'objet de publications récentes. Les recherches dans ce domaine se développent depuis quelques années, mais restent encore assez pauvres. Nous soulignons néanmoins les travaux de R. Imber (1990), P. Mariotti (1993), C. Nadelson (1974), J. Yakeley (2014) et M. Mac Garthy (1964). Cette dernière propose de considérer la grossesse comme senti: «[La grossesse est] une expérience unique pour explorer les conflits concernant la perte, les relations précoces mère-enfant, les rivalités et la sexualité [...] [S]i cela n'est pas travaillé, ce n'est pas seulement une opportunité de perdue, mais le patient pourrait agir ces sentiments non exprimés.» (Mac Garthy, 1964, p. 690) R. Imber (1964) et L. Uyehara et al. (1993) comparent l'état de grossesse chez l'analyste à une maladie importante, car, même s'il s'agit de situations tout à fait différentes, dans les deux cas, la maladie et la grossesse, le cadre de la cure va être bouleversé à cause de la situation corporelle de l'analyste. Selon Imber (1964), et 
selon les travaux de C. Nadelson (1974), la grossesse de l'analyste réactiverait en elle des ressentis d'agressivité et d'hostilité, du fait de sa plus grande vulnérabilité. Ce propos réfère à ce que D. W. Winnicott (1954) développe sous le terme de "préoccupation maternelle primaire», à savoir un processus régressif chez la femme enceinte vers un fonctionnement psychique plus primaire qui pourrait induire, chez l'analyste, des transformations quant au niveau d'écoute du matériel du patient accueilli. J. Yakeley (2014) propose une lecture de l'effet de sa grossesse comme la réactivation de problématiques liées à l'image du corps de ses patients, qu'ils soient hommes ou femmes.

C'est en grande partie ce qui va nous intéresser dans cet article: comment l'état gravide de la thérapeute peut-il contribuer à une remobilisation chez son patient de processus de séparation/individuation (Mahler, 1968) et d'identifications maternelles et féminines, processus de subjectivation précoces où les motions pulsionnelles dans leurs valences passive/active s'articulent avec la construction d'un soi différencié de l'objet? Nous serons attentifs aux motions agressives envieuses, telles que définies par M. Klein, où l'éloignement de l'objet est vécu sur un mode persécutif. Nous pourrons aussi l'entendre comme une hostilité, un sentiment d'abandon causé par l'éloignement psychique et puis physique de la thérapeute. Ces considérations nous semblent d'autant plus importantes chez de jeunes filles en train d'élaborer leur puberté. Une analyse du suivi thérapeutique d'une adolescente, Mila, nous permettra d'illustrer ces considérations théoriques, techniques et processuelles.

\section{Présentation clinique}

La thérapie de Mila a commencé lorsqu'elle était en fin de $6^{\text {e }}$, à 11 ans, et ma grossesse est intervenue alors qu'elle était en $4^{\text {e }}$, à 13 ans, soit environ après deux ans de thérapie, à raison d'une fois par semaine. Le cadre était tel qu'elle venait seule à ses séances et que je rencontrais ses parents régulièrement. Ceux-ci, même séparés, tenaient à venir ensemble. Aujourd'hui, je les reçois séparément, mais toujours avec Mila. Cette présentation clinique s'étend sur plusieurs années, ce qui permet de suivre le processus thérapeutique avec, en son sein (!), un moment de grossesse de la thérapeute. L'avant et l'après de ce moment seront compris du point de vue de l'intrication pulsionnelle de la jeune patiente et de ses mouvements identificatoires.

Lors de la première séance, le corps de Mila est perdu dans des vêtements trop larges, son visage est rond, encore enfantin, masqué par une masse de 
cheveux et de grandes lunettes, sa tête est souvent baissée. Ses parents l'entourent, visages marqués par l'angoisse. Mila sort de l'hôpital à la suite d'une tentative de suicide. Elle a été sauvée sur le fil par les pompiers qui l'ont retrouvée chez sa mère, après qu'elle ait pris les anxiolytiques de cette dernière. Mila m'est référée par la psychologue des urgences de l'hôpital.

La mère me dit que Mila ne veut jamais rien faire, qu'elle reste enfermée dans sa chambre, sur Internet. Internet qui a été sa bouée de sauvetage puisque c'est à une amie rencontrée sur un «tchat» de manga qu'elle a dit: «Je crois que j'ai fait une connerie, j'ai pris des médicaments». C'est cette jeune fille, habitant à $800 \mathrm{~km}$ et n'ayant jamais rencontré Mila, qui a contacté sa mère et appelé les urgences. La mère me dit qu'elle pense que sa fille - «leur fille» ditelle en regardant le père - doit parler de ce qui s'est passé et que sans doute il y a quelque chose qui ne va pas. Elle parle de son incompréhension, de sa culpabilité, et précise qu'elle est prête à tout entendre. L'anxiété massive de la mère envahit la pièce, étouffante au point que j'ai besoin de me tourner vers le père. Celui-ci trouve que Mila est mal depuis son entrée au collège, qu'elle est plus sombre, que cela se retrouve dans ses dessins. Je propose à Mila de dessiner si elle le souhaite. Elle le fait et représente des corps d'animaux fantastiques, des licornes, des monstres appartenant au monde de l'enfance, auxquels elle ajoute des représentations plus noires comme des cicatrices, mais aussi des attributs féminins, par exemple des crinières féminines.

Les parents de Mila se sont séparés lorsqu'elle avait 4 ans. Les soins précoces ont été donnés par ses deux parents, dont les univers émotionnels s'avèrent très différents. Les identifications primaires de Mila sont plutôt orientées par la douceur paternelle et la vigueur maternelle. Le père était dans une dynamique plus mélancolique, en deuil de son propre père. Il dit qu'il lui aura fallu beaucoup de temps pour oser penser que sa parole pouvait avoir de l'importance, le silence étant un mode de communication très installé en lui. La mère, en rupture avec son propre père, s'est identifiée de manière rigide à une fonction paternelle autoritaire et narcissiquement fragile. Sa mère est décrite comme effacée. Dans l'histoire de Mila, la figure du grand-père maternel est traumatique. La mère de Mila maintient un silence absolu sur les raisons qui l'ont conduite à rompre les liens avec celui-ci peu de temps avant de rencontrer le père de Mila. Il est même impossible de savoir s'il est mort ou pas. «Je ne veux pas en parler» est sa réponse. Elle laisse planer sur sa disparition un fantasme de mort et de violence terrifiant. Son silence intime férocement à Mila l'interdit d'exprimer de la curiosité pour le père de sa mère. 
Au début du suivi, la mère est envahie d'angoisse et de culpabilité, mais aussi d'une agressivité intense. Durant plusieurs mois après ce passage à l'acte de Mila, le risque de mort envahit ses possibilités d'accueillir l'agressivité de sa fille. La mère s'inquiète de son silence, de son côté sombre. Mila ne parle pas beaucoup directement de ses émotions, sauf par le dessin. Elle a toujours à la main de quoi dessiner. En séance, en cours, chez elle, avec ses amis, le dessin est un recours expressif salvateur. La mère veut créer avec moi une alliance paradoxale: elle m'appelle «docteur» et en même temps m'explique ce qu'est un adolescent puisqu'elle travaille comme éducatrice auprès d'eux. Elle «sait » que sa fille va mal et, effectivement, en séance Mila accentue son attitude morose, détachée de l'inquiétude de sa mère. À mes questions, elle répond vaguement, accentuant seulement les traits de clivage sur le dessin: visage masqué par des cheveux, corps chimérique...

Le suivi va se mettre en place de manière très régulière avec d'emblée des transferts multiples des parents et de Mila. De manière intuitive puis plus confirmée, je propose un espace bien différencié pour Mila dont l'organisation psychique est encore extrêmement défensive avec un moi très fragilisé par la brutalité de la survenue pubertaire et l'entrée au collège. De plus, elle se protège de l'intensité de l'angoisse maternelle et ne peut pas tout à fait trouver chez son père un contre-pied tiercéisant.

Les premières séances avec Mila s'engagent sur une tonalité de discussion portant sur des objets de médiation comme les mangas et des figures mi-animales, mi-humaines surtout. Elle mêle un univers très enfantin et tendre, voire naïf, et des émergences sombres, gores. Peu à peu ses dessins se sexualisent et les personnages prennent des formes féminines. Ses relations avec les autres sont au centre de ses préoccupations. Elle cherche des points identificatoires avec les filles de son collège, à partir de détails sensoriels comme la couleur bleue des cheveux de l'une ou la tenue noire de l'autre. Les tatouages et les marquages ont fonction identitaire, car ils lui permettent de catégoriser les personnes. Les uns sont les «goth», les autres les «kawaï, ou les métalleux». Le signe fait identité et Mila observe avec envie la certitude affichée de ses amis. Elle-même va se teindre les cheveux, porter des colliers cloutés ou encore des vêtements d'animaux «mignons». Elle témoigne par son style de ses vécus contrastés entre tendresse exacerbée et désespérée et mouvements agressifs, en elle et avec les autres.

Après quelques mois de séances, Mila présente des aspects changeants dans son corps: elle vient en séance avec un écarteur à l'oreille qu'elle cache sous ses cheveux. Je suis la première adulte à qui elle le montre, ses parents ne 
le savent pas encore. Elle est en $5^{e}$ et c'est un choc pour ses parents lorsqu'ils le découvrent. Avec moi en séance, elle teste d'autres relations maternelles et paternelles, me mettant parfois en rivalité avec ses parents. Vais-je être choquée aussi? Vais-je être séduite? De plus en plus, Mila exprime son désir de différenciation, se confronte à sa mère et utilise l'espace thérapeutique comme lieu transitionnel. Le trou de la peau de Mila, son piercing, semble lui permettre d'énoncer une lacune de symbolisation dans le mouvement de séparation d'avec sa mère; elle le subjective dans la séance par son jeu de cache-cache entre ses parents et moi. Au bout d'une année de travail, les relations entre Mila et sa mère deviennent très agressives. Mila se renferme dans le silence lorsqu'elle est chez celle-ci et sa mère le vit à la fois comme un danger dépressif et comme une attaque de leur lien affectif. La mère a l'impression que sa fille ne l'aime pas et ne demande que de l'argent. Mila se sent étouffée par les demandes de reconnaissance affective de sa mère qui lui écrit de longues lettres où elle décrit sa souffrance d'avoir une fille aussi indifférente. Mila demande à vivre chez son père, décision qui sera discutée en séance et qui causera chez la mère une importante blessure narcissique.

Sa fille veut la forcer à parler du grand-père énigmatique, mais la mère refuse. Mon ressenti contre-transférentiel est massif: je m'identifie à la mère en tant que fille murée dans un silence face à son père et qui, devant l'injonction de sa propre fille, est démunie. Je comprends aussi l'appel exigeant de Mila auprès de sa mère, car elle-même s'est identifiée à ce grand-père qu'elle imagine maltraitant, comme elle: "Je suis toxique, je le vois bien, je fais du mal à ma mère.» Leur relation en miroir projectif ne permet pas que l'une et l'autre s'émeuvent sans se blesser. La mère hurle, part en claquant la porte, mais me rappelle dans la journée pour parler de sa détresse, de son incompréhension. L'espace de la séance, lui-même écho des mouvements psychiques entre Mila et ses parents, doit être souple: je dois travailler avec chacun un espace psychique pour qu'il soit aussi possible de se penser ensemble. Lorsque Mila demande à vivre chez son père, elle a recours à lui comme contenant pour construire une distance nécessaire pour protéger les bons aspects de l'objet maternel. Ainsi, le lien mère-fille, plus froid, clivé de manière fonctionnelle, permet néanmoins qu'il survive.

C'est dans cette configuration familiale et après presque deux ans de thérapie que ma grossesse intervient. L'investissement de la thérapie par Mila, mais aussi par les parents m'amène à réfléchir à l'accueil de l'annonce de mon état. Comment Mila va-t-elle réagir alors qu'elle a tellement de difficulté à construire un discours sur ses éprouvés primaires ou encore sur 
ses relations avec ses parents? Comment vont réagir les parents à l'annonce de mon futur départ et au fait que j'ai choisi d'investir un autre enfant que le leur? Je choisis d'attendre pour annoncer ma grossesse, car, même si la taille de mon ventre la rend plus visible, $\mathrm{j}$ 'attends l'occasion d'un écho de cette situation dans le transfert. Mon inquiétude porte à la fois sur l'aspect envahissant de la rivalité narcissique avec cet autre enfant et sur le vécu d'abandon lors de mon départ. De plus, la fragilité de Mila concernant ses identifications féminines et maternelles a pu amener dans le transfert des éléments d'homosexualité intenses. Ses identifications féminines et masculines restent troubles et son sentiment de continuité identitaire encore plus. C'est surtout cet aspect de son organisation psychique qui m'amène à la prudence dans mes interventions.

En séance, le dessin est devenu une zone de contact ritualisée entre elle et moi. Je lui fournis en début de séance un support et du papier; elle parle en dessinant, soit de manière quasi automatique, donnant à son dessin le fil d'un rébus, soit de manière beaucoup plus appuyée, surtout lorsqu'elle s'interroge sur des mouvements intérieurs. Par-delà la feuille, son regard est furtif, mais la présence de Mila est très habitée. Sur le plan transférentiel, on pourrait considérer la feuille de papier et son support comme un toucher entre elle et moi. Je suis émue par cette jeune fille au verbe haut, à l'humour décalé qui laisse transparaître une fragilité farouchement défendue. Le contact émotionnel et tactile doit passer par une codification très stylisée, de mots adolescents, de gestes-signes, sans quoi elle se sent envahie, débordée. Ce qui se joue entre elle et moi constitue un appui sur la relation et permet l'émergence de différentes identifications, différentes facettes de la jeune fille qu'elle devient. Mila présente une image d'elle floue. Son visage est maquillé, apprêté d'attributs gothiques, ses cheveux sont teints, en bleu, en violet. Son corps en revanche est souvent dissimulé sous d'amples sweat-shirts ou bien sous un treillis de son père. Ses vécus persécutifs sont présents lorsqu'elle parle de ses amis et particulièrement de ses relations amicales féminines: «Elle me prend mes idées, mes pensées, mais c'est à moi.» De même, son unique relation amoureuse, homosexuelle, est vécue de manière distancée: «Elle m'aimait tellement que du coup quand elle m'a demandé d'être avec elle j'ai dit ok... finalement c'était pas une bonne idée, je l'aimais pas.»

Sa tête est emplie de choses enviables, mais qui la laissent vide; le dedans soutient le dehors et réciproquement, dans une dynamique de retournement non limitée par la castration œdipienne. C'est à elle ou à l'autre, et si aimer veut dire projeter, est-ce se perdre? Comment aimer sans (dé) posséder? Son 
questionnement identitaire, identificatoire, laisse de côté les aspects masculin/féminin, et la bisexualité est source d'angoisse et pourtant s'exprime beaucoup, notamment par les dessins. Elle représente surtout des femmes, aux formes plus marquées par le genre, même si toujours chimériques dans leurs attributs. Il s'agit de femmes-licornes, de femmes-chats. La différence des genres est encore représentée sous forme mi-humaine, mi-animale.

Si au début de ma grossesse il n'est pas du tout question de maternité ni de bébé, à partir du $5^{\mathrm{e}}$ mois, le contenu des séances devient de plus en plus orienté vers les bébés: "J'ai un petit frère, enfin pas vraiment, c'est le fils de la copine de mon père, mais c'est marrant, du coup je m'amuse avec lui, il est cute.» Rien n'avait encore été verbalisé concernant mon état. Grâce à ce rêve cela fut possible:

C'est chelou, en ce moment je sais pas ce que j'ai, mais je rêve de bébés. La nuit dernière j'ai rêvé d'un bébé, mais il était là, posé devant moi et il avait trois yeux, un autre dans le front. Quand je m'approche en fait je rentre dans son œil, (mime le geste d'être happée) là celui de la tête et c'est trop beau, on voit les galaxies, les étoiles... tout l'univers... C'est pas un bébé, c'est comme un Bouddha...

L'allusion est tellement nette avec la présence de ce bébé énigmatique pour lequel elle a une curiosité féroce, mais silencieuse, que je lui dis que je suis enceinte et qu'elle ne sait rien de ce bébé. Elle répond qu'elle se demandait, mais n'osait pas en parler. Le regard unique et omniscient du bébé du rêve fait penser à une projection de son propre regard sur le visage de la mère. Une vision qui n'est pas binoculaire, mais où l'objet est entièrement vu selon un seul angle. Cette façon de voir correspond également à celle du tout jeune bébé qui n’a pas encore la possibilité de relier les deux images de ses yeux. Pour Mila, cet ancrage somatique fait le lit d'une difficulté à intérioriser une scène primitive bisexuelle où père et mère forment un objet interne fondamental pour la pensée. Le «Bouddha» est aussi métaphoriquement cette figure omnisciente et immortelle, occultant les envies et angoisses de mort autour de ce bébé-dans-le ventre-tête de la thérapeute. Il est aussi un corps rond, replet, un bébé avec beaucoup de plis. Je dis "ventre-tête», car par cet œil et l'importance du regard pour Mila, il semble qu'il peut y avoir des confusions de zones (Meltzer, 1992) entre la tête et le ventre.

J'insiste ici sur les aspects sensoriels, formels, de ce bébé, car c'est avec une curiosité étonnée que Mila me le décrit et qu’elle insiste pour que je vive 
avec elle cet étonnement. Partager une même vision, l'éprouver ensemble, n'est-ce pas un besoin d'être reconnue comme semblable? L'étonnement partagé de la survenue de ce Bouddha peut s'entendre comme l'étonnement de la survenue pubertaire, arrivant par surprise, exigeant en elle ses capacités psychiques à penser le corps, ses sensations, ses fantasmes, mais aussi l'étonnement de cette grossesse, survenue sans qu'elle puisse s'y attendre. Dans ce rêve se trouvent également des fantasmes correspondant à un registre primaire, comme l'envie de me pénétrer, au sens d'une possession envieuse (Klein, 1957) de l'intérieur de mon corps. Mila témoigne ainsi de ses identifications à la fois au bébé, mais aussi au contenant de celui-ci, une "galaxie». Le dedans et le dehors sont dans une réversibilité correspondant là encore à des fantasmes régressifs.

Un aspect contre-transférentiel important dans cette séance est la conscience accrue que j'ai de l'enveloppe que représente mon ventre pour mon bébé. Est-ce une position défensive due à ma sensibilité régressive pour mon enfant? J'accueille sur le moment cette pensée comme étant également une identification projective au besoin de Mila d'avoir des contenants psychiques qui communiquent entre eux de manière transitionnelle et non clivée. M’identifiant plus au bébé, je ressens de manière plus accrue son besoin de protection, l'enveloppe et la transformation sensori-affective. Ainsi, par mon contre-transfert, $j$ 'accompagne Mila dans la traversée régressive de ses propres limites intersubjectives.

Pour Mila l'annonce de ma grossesse est distincte de la séparation. La première est liée à l'apaisement de sa curiosité: elle doutait que je sois enceinte et n'osait demander. La seconde est contenue par mon énonciation de mon souci de continuité, alors qu'elle exprime défensivement avec humour son angoisse de séparation: «Bon, c'est pas comme si vous disparaissiez, vous allez revenir après.»

Du côté des parents, l'annonce de ma grossesse ne suscite pas d'expression de sympathie, mais plutôt une inquiétude concernant le suivi de Mila. Après un mouvement de vexation narcissique, j'entends mieux à quel point il m'est demandé de faire taire tout désir subjectif, à quel point la peur de l'effondrement est présente. Je mets en place un dispositif d'accueil et de continuité durant mon absence en impliquant une collègue de mon cabinet. Je pars en congé de maternité avec une inquiétude liée à ma culpabilité de laisser mes patients, un reste affectif qui me permet par ailleurs de rester en lien avec eux. La subjectivation de la séparation est décidément difficile et c'est grâce à la grossesse que je peux la percevoir de manière si sensible. 


\section{Absence de corps}

Alors que je suis en congé de maternité, la mère m'appelle de nombreuses fois et, face à son insistance, je finis par répondre. Habituellement, nous nous voyons seulement en séance et ses appels sont donc hors cadre. Elle m'annonce qu'elle a un cancer du sein, mais qu'elle ne sait pas comment le dire à sa fille, ni où elle doit effectuer son traitement. À quelle place transférentielle suis-je pour la mère si mon absence est remplie par ce cancer du sein? Est-ce mon absence ou bien les séances de thérapie de sa fille qui produisent un vide représentationnel? Est-ce un écho de sa propre rupture familiale? Le corps absent, menaçant, serait-il incorporé comme un objet toxique, le cancer du sein? La mère de Mila énonce des craintes concernant l'effondrement de sa fille face à son cancer ainsi qu'un nouveau passage à l'acte suicidaire. J'entends plusieurs mouvements dans cette plainte. Je perçois, impuissante, son désarroi face à la symbolisation des sensations liées à son deuil d'un environnement maternel soutenant. J’y entends également le souhait que sa fille soit atteinte émotionnellement par sa maladie, comme une attente désespérée de preuve d'attachement. En même temps, elle refuse l'expression de mon empathie envers elle: «Ça va moi, j'ai pas besoin d'aide, c'est Mila qui m'inquiète, je suis sa mère quand même ça va être dur pour elle.» La tendresse est à la fois souhaitée et redoutée. C'est donc par téléphone que j'accompagne cette mère, alors que ma collègue reçoit une fois par mois Mila et a aussi reçu les parents.

\section{Retrouvailles}

Au retour du congé de maternité, j'observe chez Mila une modification de ses investissements d'objets sexuels: le matériel porte sur des hommes et des femmes. Ces hommes ont comme spécificité d'avoir une curiosité ingénieuse. Ainsi elle parle de Sherlock Holmes comme d'un homme sexy et intelligent: «Il sait résoudre des énigmes comme ça et il est class. Et puis il dit les choses de manière précise, il est très observateur... tout comme moi! Je sais pas si c'est mon double ou mon homme idéal!» Par ailleurs, elle exprime une relation à sa mère plus complice, où elles regardent ensemble des séries de morts-vivants et où le rire et l'humour leur permettent de se jouer de leurs angoisses de mort. Alors que Mila avait demandé à réduire son temps de présence chez sa mère, elle reprend un rythme équitable chez ses deux parents. Est-ce que mon absence a permis à Mila de réinvestir l'espace ventre maternel? «On mange des sushis ou des tacos avec maman», dit-elle.

Je m'interroge sur la perte de contenance vécue par la mère lors de mon congé maternité et, par là, de l'utilisation par Mila de l'espace thérapeutique 
comme faisant fonction d'une peau psychique différenciée des aspects adhésifs à sa propre mère. Mila a besoin de représenter par le dessin des sensations internes inquiétantes et étranges, des mouvements qu'elle ne sait pas reconnaitre comme siens et qui la font se sentir persécutée. Malgré ces éléments d'un sein nourricier maternel, le clivage du sein toxique reste présent. En effet, Mila ne sait pas comment l'autre va recevoir l'amour, la tristesse et la dépendance qu'elle peut éprouver. Lorsque ses amis lui témoignent un investissement affectif, elle est excitée et se défend par une mise à distance quasi phobique de l'objet.

Petit à petit, elle peut ressentir le regard des autres. Ainsi, elle rencontre des garçons avec lesquels elle se sent regardée comme une fille. Durant un voyage scolaire avant l'été, elle s'ouvre à des relations amoureuses fantasmées avec des garçons de son âge, s'étonnant que cela soit si simple pour les autres de la voir comme fille. Durant l'été, elle nouera des liens avec un garçon rencontré sur Internet, qu'elle rencontrera en personne, accompagnée par sa mère. La réalité et le virtuel se nouent de manière plus sécurisée. Ainsi, l'intrication d'un lien primaire homosexuel permet à Mila d'autres formes de liens érotiques. Toutefois, son image du corps reste fragile et elle traverse des moments où elle le ressent, devant se protéger par un enfermement et un isolement face aux sollicitations extérieures. Ainsi, après quelques semaines de reprise des cours, elle passera un week-end enfermée dans sa chambre, comme pour rassembler des aspects épars d'elle-même, ce qu'elle exprime ainsi:

J'ai besoin de ma grotte, c'est ma petite dépression, j'ai besoin qu'on me fiche la paix, plus rien entendre. En fait la vie elle est trop brutale, les gens ils sont pas cool. Parfois je sais pas s'ils sont méchants ou pas. Moi je suis née et j'étais pas prête, je dormais quand le médecin il m’a sortie du ventre de ma mère. C'est vrai, j'étais là, toute tranquille en train de dormir et cette grosse main vient me réveiller! J'avais rien demandé moi.

Cette association révèle le fantasme d'une intrusion primaire, mais est aussi la métaphore d'un débordement pulsionnel. Durant ce week-end, elle a fait une œuvre représentant un sexe féminin sanguinolent, percé. En effet, son corps, effracté par la puberté, est en inadéquation avec ses capacités de contenance. Durant les quelques séances précédant la date anniversaire de mon départ en congé maternité (donc un an après), elle parle beaucoup de piercings, autorisés ou non par sa mère. Les termes employés sont vifs, crus, 
comme des objets partiels morcelés. Durant cette période, elle dessine des femmes cyclopes. Ainsi, le troisième œil du bébé-Bouddha se retrouve sur le visage de ces femmes, mais il est seul. Ces femmes semblent devenir des objets combinés, confus entre le bébé et la mère, aplatissant les enveloppes permettant l'identité et l'individuation. L'association du bébé aux trois yeux, de la reconstruction de sa naissance et de ces femmes cyclopes coïncide avec la date anniversaire de mon départ. J'exprime à Mila cette coïncidence. Je choisis des mots neutres dans le contenu, mais je les associe à la surprise que j'éprouve, en écho à l'étonnement que nous avions partagé lorsqu'elle m'avait raconté son rêve. Elle sourit, s'apaise et se rappelle cette période. Il semble que le rassemblement temporel apaise les angoisses d'effraction corporelle. En effet, dès la séance suivante, la femme cyclope a deux yeux, dont un qu'elle cligne avec humour. L'ouverture narrative est de nouveau possible.

Alors qu'aujourd'hui Mila a 14 ans et qu'elle est en $3^{\text {e }}$, elle doit décider de son orientation scolaire. Elle souhaite faire un bac professionnel tandis que sa mère refuse. Pour la première fois elle exprime clairement sa détresse face à un élan qu'elle ressent réprimé de manière violente. Elle commente: "C'est comme un bébé, quand il a envie de quelque chose, mais qu'il a peur il se tourne vers sa mère et lui tend les bras. Si la mère elle lui dit non, et qu'elle n'est pas là, lui il tombe.» Il est rare qu'elle commente aussi directement ses états émotionnels et qu'elle me permette de les partager. Émue et envahie d'une tristesse sensorielle, je sens les larmes en moi. Elle tourne en dérision sa réaction, mais lorsque je verbalise son émotion, elle dit que c'est dur, son ton est grave. Elle ajoute: «Vous savez, moi je perds plus mes cheveux que ma mère, elle, elle a eu quelques rayons donc elle perd ses cheveux, moi c'est par poignées qu'ils tombent. J'en fais des petites boules...» Elle met sa capuche autour de sa tête, son sweat-shirt ne laisse dépasser d'elle qu'un visage sans émotion, interrogatif et inquiétant; un bébé Bouddha sombre. Je lui dis que c'est différent ce qui se passe pour sa mère et elle; pourtant, elle se fait beaucoup de souci alors qu'elle a l'impression que personne ne pense à son souci à elle. Je m'interroge sur ses aspects bébés, ceux où elle s'est identifiée à des visages inquiétants, sans portage psychique ou physique. La tête-sein de Mila est bien entravée par des aspects mortifères contre lesquels elle lutte par une créativité forcenée.

Néanmoins n'est-ce pas un pas vers la subjectivation que de pouvoir associer toute l'ambivalence de ce bébé-Bouddha, tant du côté de l'avenir que des angoisses et des vœux de mort? Elle a pu s'identifier dans le transfert 
à la mère d'un bébé, à une femme généralisée, mais aussi à un bébé étrange, vivre des expériences régressives et se retrouver après une séparation.

\section{Réflexions théoriques}

C. Néri décrit la contenance psychique en ces termes: «La contenance peut être définie comme une modalité à travers laquelle sont tenus ensemble des parties ou des fragments, ou encore comme un processus à travers lequel prend forme et consistance quelque chose qui auparavant était complètement informe.» (Néri, 2011, p. 66) Je pense que pour Mila le processus thérapeutique et la grossesse ont eu une fonction de contenance dans le processus de régression formelle, car elle a pu vivre de nouveau une attention proche de ses besoins primaires: une attention à ses sensations, à son corps, à ses confusions. L'appui sur la rythmicité du cadre a servi de tiers et a participé à une narrativité continue, étayage à l'élaboration de sa propre continuité d'être. D'autre part, l'exploration d'aspects bébés d'elle-même s'est appuyée sur l'identification au bébé présent dans mon ventre pendant ses séances, ce tiers impromptu qui a également servi de support projectif. En utilisant les séances comme une enveloppe pare-excitante vis-à-vis des attaques envieuses de sa mère, elle a pu accentuer cette rivalité en décrétant qu'elle n'avait pas besoin de lui parler, car elle m'avait, moi, exprimant ainsi le clivage entre le bon et le mauvais sein. De mon côté, j'ai appris à accueillir les aspects "pénétrants» de la mère, tout en m'identifiant à l'appel de la tendresse chez Mila.

Un autre aspect de compréhension de la remobilisation psychique de Mila est son image du corps, son auto-érotisation et ses objets libidinaux. Des parties de son corps sont très investies, comme les yeux, les oreilles et les cheveux, mais le reste est assez indifférencié, du moins au début du suivi. Mila cherche une image à laquelle elle pourrait s'identifier et ce mouvement est tellement archaïque qu'il se confond avec l'objet d'amour. Il y a un retournement permanent de son sentiment de soi vers l'objet, y compris dans son vécu de l'homosexualité. Être fille équivaut à aimer les filles. Quand une copine lui dit qu'elles sont en couple, alors Mila accepte sans que cela fasse écho à un désir autre que celui d'être en double, comme pour étayer son propre sentiment d'identité. Le dedans et le dehors, l'objet et le sujet s'entremêlent et sont plus organisés par une logique narcissique qu'œedipienne. Par la réalité de la scène gravide, où dans la séance un objet était contenu par un contenant, elle s'est peut-être identifiée à la possibilité de penser à la fois le dedans et le dehors sans exclusion de l'un ou de l'autre. 
Nous pensons que cette projection identificatoire de Mila à fois au contenant-mère et au contenu-bébé s'est peu à peu organisée par le mouvement au sein des séances et par leur intrication.

La transformation d'un transfert envers une thérapeute femme vers une thérapeute mère s'est faite dans le silence au début: "Je m’en doutais, mais j'étais pas sûre», dira-t-elle. Je pense que mon changement d'état corporel vers celui de femme-portant-un-univers-en-elle - j'utilise cette formule pour souligner que c'est la croyance en sa propre créativité interne de vie (Winnicott, 1954) qui permet d'asseoir un sentiment d'exister plus stable et un appui sur de bons objets internes. G. Haag (2006 a, 2006 b) parlerait d'un «fond de sécurité» fait d'un objet d'arrière-plan et d'une interpénétration des regards. Ces deux mouvements - la créativité et le fond de sécuritése répondent et s'entendent au niveau temporel dans un récit en après-coup possible. Ainsi, à partir du matériel de Mila, l'interprétation de la répétition de son rêve avec le «souvenir» de sa naissance (soit la première séparation d'avec sa mère) un an après en est une illustration. Sa curiosité à l'égard de ce bébé en séance lui a permis d'effectuer un «travail du rêve» qui traduit bien le bouleversement de la découverte de l'immensité du contenant maternel, cet «objet» qui anime une pulsion épistémophilique: est-ce aussi beau dedans que dehors? D. Meltzer dira à ce sujet: «Ce que j'appelle le "conflit esthétique", c'est la douleur qui vient de l'incertitude due à la juxtaposition de ce qui peut être observé de l'extérieur de l'objet et de ce qui n'est pas observable, l'intérieur de cet objet.» (Meltzer, 2013, p. 36) Découvrir qu'il y a une limite franchissable entre le dedans et le dehors est vertigineux, dangereux pour le jeune bébé. Lorsque Mila rêve d'un bébé dont l'exploration le mène aux confins de l'univers, la beauté de celui-ci n'enlève rien à l'inquiétude de son infinitude. La galaxie et ses étoiles sont comme des promesses liées à la rencontre de l'intérieur du corps de la mère.

Dans le rêve, le mouvement est princeps: Mila le décrit comme une projection au travers du corps du bébé. Sur le plan temporel, ce mouvement qui la happe se retrouve dans le surgissement du souvenir, un an plus tard, de ce même rêve. Entendre la répétition a une fonction contenante car elle offre à Mila la possibilité d'une trame narrative à partir de l'histoire transférentielle. La fragilité de son sentiment d'exister est tangible: se dessiner n'est pas seulement s'exprimer, mais aussi essayer de s'assurer d'une certitude d'exister, pour un autre qui la voit et la regarde se découvrir. Or, ce bébé échappe à sa vision et celui-ci est vécu par elle avec inquiétude: c'est ici que l'énigme est éprouvée. Nous retrouvons l'hypothèse de P. Gutton (2009), 
qui dit que des adolescents ont d'abord besoin d'être reconnus avant d'être compris. Autrement dit, il faut d'abord s'assurer d'un sentiment de soi pour pouvoir tolérer les aspects non compréhensibles. Nous avons là encore une autre expression d'intrication d'éléments féminin et masculin (contenant/ contenu) au sens primaire, mais aussi, nous allons le voir, au sens génitalisé. Elle découvre qu'il existe des objets qu'elle peut investir sans les détruire par envie, et ce, malgré le fait qu'ils lui échappent, malgré le fait qu'ils soient plus complexes que ce qu'elle peut maîtriser d'eux.

Mila dit qu'elle n'aime pas les bébés, car leur dépendance absolue l'angoisse trop. Éprouver sans crainte le lien régressif à un autre est très difficile, mais elle s'y autorise par petites touches dans l'espace thérapeutique. Le fait que, pendant qu'elle me parle de son rêve, je sois plus sensible aux contenants de mon corps qu'aux mouvements internes du bébé réel, me semble un outil pour comprendre la nécessité pour Mila de faire taire ses propres sensations de vide anaclitique. C'est par le contre-transfert sensoriel auquel je peux être attentive, en silence, mais en écoute, que nous apprenons, au sens d'un processus d'apprentissage par l'expérience, l'existence d'un besoin de renforcement des limites entre soi et l'autre.

Un dernier point est à entendre: il s'agit de l'intrication des éléments sensoriels précoces avec ceux génitaux. En effet, n'oublions pas que même si Mila ne l'évoque pas directement au moment où j'énonce un lien entre son rêve et ma grossesse, il y a un homme derrière celle-ci. Il ne s'agit pas seulement d'un objet partiel, tel le fantasme décrit par M. Klein du pénis dans le ventre de la mère, mais aussi du fantasme d'une scène primitive, dont elle méconnaît les secrets et qui anime ainsi sa curiosité, donc sa pensée créative. À mon retour de congé maternité, j'entends cette ouverture dans son identification à Sherlock Holmes, un homme curieux et inventif. Ainsi, elle aussi peut reconnaître en elle des éléments bisexuels qui ne privent pas sa tête de son intelligence et de son humour, et, en même temps, elle peut vivre avec sa mère des moments de partage: elles mangent ensemble en regardant des séries télévisées.

\section{Conclusion}

Le travail sur les effets de l'état gravide de la thérapeute au cours du processus thérapeutique avec une adolescente, Mila, nous a permis de poser quelques hypothèses sur le double mouvement régressif qui remobilise les enveloppes primaires en deçà du verbal. Nous avons pu approcher ce mouvement par l'implication sensori-motrice dans l'écoute transféro 
-contre-transférentielle. Alors que la jeune fille doit effectuer un travail du pubertaire, elle élabore ses identifications sexuées depuis leurs registres les plus précoces jusqu'à leur génitalisation. Or, l'état mixte de sa thérapeute enceinte permet un jeu projectif sur les enveloppes, les identifications féminines, maternelles et bébés, autrement dit la question de la différenciation entre soi et un objet pas encore bien constitué. Pour F. Guignard (1996) l'espace maternel est le socle psychique à partir duquel se construit l'espace féminin, espace de la bisexualité. Le socle maternel s'est appuyé pour une partie sur un dépôt dans le cadre des séances des aspects les moins élaborés d'elle-même, ainsi que sur une écoute contre-transférentielle par le corps. Le féminin s'inscrivait dans toutes les séparations rythmées et particulièrement dans celle du congé maternité.

Nous avons pu mettre en lumière la force des fantasmes envieux de la patiente et de sa mère à l'égard d'un contenant maternel, représenté par les séances de thérapie. L'élaboration de ces fantasmes a pu conduire à une intrication pulsionnelle plus large, intégrant également l'ambivalence liée à la séparation d'avec l'objet. Nous avons vu que la qualification de l'objet était nécessaire pour sortir de la confusion entre une dépendance primaire et un élan d'individuation psychique propre au processus adolescent. Le risque d'effacement de l'objet lors de cette transformation qualitative devait être contenu psychiquement et la grossesse en a été, en partie, un support projectif. En effet, nous avons entendu à la fois des fantasmes archaïques touchant à l'intégrité corporelle, mais aussi à la construction d'un sentiment de soi ayant recours à plus de transitionnalité psychique avec des croisements de registres sensoriels, figuratifs et verbaux.

Le mouvement, tantôt entravé, tantôt projectif, ressenti dans le corps et dans les départs-retrouvailles entre les séances, s'avère central dans le processus thérapeutique. M. Fain et P. Marty (1954) pensent que la motricité constitue l'une des premières relations d'objet qui perdure chez l'être humain sous des formes plus secondarisées. Peut-être que le mouvement psychique de la thérapeute enceinte, le mouvement d'un corps qui croît, permet de mettre au travail des aspects corporels peu subjectivés, pour peu que l'on y porte une attention sensible et tiercéissante.

Anne Boisseuil aboisseuil@gmail.com 


\section{Références}

Bleger, J. (1979). Psychanalyse du cadre psychanalytique. Dans R. Kaës (dir.), Crise, rupture et dépassement (p. 255-285). Paris: Dunod, 2004.

Ferenczi, S. (1931). Lettre 1201; 15 septembre 1931. Dans Correspondance Freud-Ferenczi (1920-1933). Paris: Calmann-Lévy, 2000.

Freud, S. (1904). La méthode psychanalytique. Dans La technique psychanalytique. Paris: Presses universitaires de France, 1953.

Freud, S. (1911). Formulation sur les deux principes du cours des évènements psychiques. Dans Résultats, idées, problèmes, I. Paris: Presses universitaires de France, 1984.

Golse, B. et Roussillon, R. (2010). La naissance de l'objet. Paris: Presses universitaires de France.

Guignard, F. (1996). Au vif de l'infantile. Lausanne: Delachaux et Niestlé.

Gutton, P. (2009). La cure de l'adolescence entre biographie et construction. Topique, 3 (108), $37-46$.

Haag, G. (2006 a). Clinique psychanalytique de l'autisme et formation de contenance. Dans A. Green (dir.), Les voies nouvelles de la thérapeutique psychanalytique: le dedans et le dehors (p. 600-628). Paris: Presses universitaires de France.

Haag, G. (2006 b). Clivage dans les premières organisations du moi: sensorialités, organisation perceptive et image du corps. Carnet Psy, 8 (112), 40-42.

Imber, R. (1964). The avoidance of countertransference awareness in a pregnant analyst. Contemporary Psychoanalysis, 26, 223-236.

Klein, M. (1932). La psychanalyse des enfants. Paris: Presses universitaires de France, 1959.

Klein, M. (1957). Envie et gratitude et autres essais. Paris: Gallimard, 1968.

Mahler, M. (1968). Psychose infantile. Paris: Payot, 1982.

MacGarthy, M. (1964). The analyst's Pregnancy. New York: NY Academic Press.

Marty, P. et Fain, M. (1954). Importance du rôle de la motricité dans la relation d'objet. Revue française de psychanalyse, 19 (1-2), 205-284.

Mariotti, P. (1993). The analyst's pregnancy: The patient, the analyst and the space of the unknown. International Journal of Psychoanalysis, 74, 151-64.

Meltzer, D. (1992). La dimension géographique de l'appareil psychique. Le Claustrum. Larmor-Plage: Hublot.

Meltzer, D. (2013). Sur l'objet et le conflit esthétique. Journal de la psychanalyse de l'enfant, $3(1), 29-39$.

Nadelson, C., Natman, M., Arons, E. et Feldman, J. (1974). The pregnant Therapist. American Journal of Psychiatry, 131, 1107-1111.

Néri, C. (2011). Le groupe: Manuel de psychanalyse de groupe. Paris: Broché.

Uyehara L. A. et al. (1993). Telling about the analyst's pregnancy, JAPA, 43(1), 113-135.

Yakeley J. et al. (2014). Voir, refléter, désirer: l'impact du corps de l'analyste enceinte sur l'image corporelle du patient, L'année psychanalytique internationale, 1, 17-43.

Winnicott D. W. (1954). La préoccupation maternelle primaire. Dans De la pédiatrie à la psychanalyse (p. 285-292). Paris: Payot, 1992. 